EPJ Web of Conferences 64, 04003 (2014)

DOI: $10.1051 /$ epjconf/ 20146404003

(C) Owned by the authors, published by EDP Sciences, 2014

\title{
3D numerical modeling of YSO accretion shocks
}

T. Matsakos $1,2,3, a$, J.-P. Chièze ${ }^{2}$, C. Stehlé ${ }^{3}$, M. González ${ }^{4}$, L. Ibgui ${ }^{3}$, L. de Sá ${ }^{2,3}$, T. Lanz ${ }^{5}$, S. Orlando ${ }^{6}$, R. Bonito ${ }^{7,6}$, C. Argiroffi ${ }^{7,6}$, F. Reale ${ }^{7,6}$, and G. Peres ${ }^{7,6}$

${ }^{1}$ CEA, IRAMIS, Service Photons, Atomes et Molécules, 91191 Gif-sur-Yvette, France

${ }^{2}$ Laboratoire AIM, CEA/DSM - CNRS - Université Paris Diderot, IRFU/Service d'Astrophysique, CEA Saclay, Orme des Merisiers, 91191 Gif-sur-Yvette, France

${ }^{3}$ LERMA, Observatoire de Paris, Université Pierre et Marie Curie and CNRS, 5 Place J. Janssen, 92195 Meudon, France

${ }^{4}$ Université Paris Diderot, Sorbonne Paris Cité, AIM, UMR 7158, CEA, CNRS, 91191 Gif-sur-Yvette, France

${ }^{5}$ Laboratoire Lagrange, Université de Nice-Sophia Antipolis, CNRS, Observatoire de la Côte d'Azur, 06304 Nice cedex 4, France

${ }^{6}$ INAF - Osservatorio Astronomico di Palermo, Piazza del Parlamento 1, 90134 Palermo, Italy

${ }^{7}$ Dipartimento di Fisica e Chimica, Università degli Studi di Palermo, Piazza del Parlamento 1, 90134

Palermo, Italy

\begin{abstract}
The dynamics of YSO accretion shocks is determined by radiative processes as well as the strength and structure of the magnetic field. A quasi-periodic emission signature is theoretically expected to be observed, but observations do not confirm any such pattern. In this work, we assume a uniform background field, in the regime of optically thin energy losses, and we study the multi-dimensional shock evolution in the presence of perturbations, i.e. clumps in the stream and an acoustic energy flux flowing at the base of the chromosphere. We perform 3D MHD simulations using the PLUTO code, modeling locally the impact of the infalling gas onto the chromosphere. We find that the structure and dynamics of the post-shock region is strongly dependent on the plasma-beta (thermal over magnetic pressure), different values of which may give distinguishable emission signatures, relevant for observations. In particular, a strong magnetic field effectively confines the plasma inside its flux tubes and leads to the formation of quasi-independent fibrils. The fibrils may oscillate out of phase and hence the sum of their contributions in the emission results in a smooth overall profile. On the contrary, a weak magnetic field is not found to have any significant effect on the shocked plasma and the turbulent hot slab that forms is found to retain its periodic signature.
\end{abstract}

\section{Introduction}

In the magnetospheric accretion scenario of young stars, material from the inner parts of the surrounding disk is guided along the field lines of the stellar magnetosphere and hits the surface with a free-fall velocity (see contributions from Kurosawa, Lii, Pudritz, Romanova, Zanni, this volume). Upon impact, a strong shock forms that heats the plasma at temperatures of a few million Kelvin. The

a e-mail: titos.matsakos@cea.fr

This is an Open Access article distributed under the terms of the Creative Commons Attribution License 2.0, which permits unrestricted use, distribution, and reproduction in any medium, provided the original work is properly cited. 

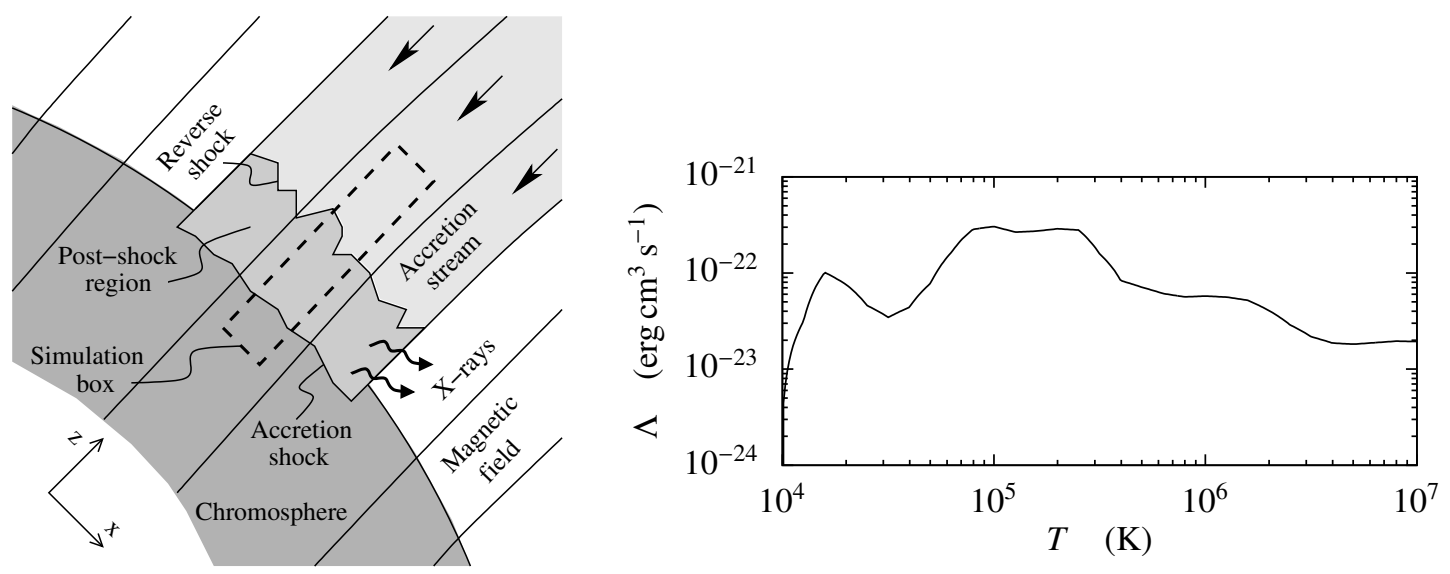

Figure 1. Left panel: a simplified sketch of an accretion shock for a strong, uniform magnetic field. Right panel: the prescribed cooling function $[4,5]$.

associated X-ray emission, which has been observed in a few protostars [e.g. 1], can provide valuable information for the structure and evolution of the post-shock region.

Theoretical 1D numerical studies [2,3] indicate that the reverse shock is expected to show a quasi-periodic behavior (see contribution from de Sá, this volume). In more detail, consider the configuration depicted in the left panel of Fig. 1 which assumes a locally uniform magnetic field. As material accumulates from the infalling gas, the post-shock region increases in height. However, the hot slab is susceptible to optically thin radiation losses with a typical cooling time of 10 to $1000 \mathrm{~s}$. This leads to a pressure drop and in turn the collapse of the reverse shock, because the infalling gas can no longer be supported. The formation-collapse cycle of the hot slab is then repeated, i.e. the reverse shock follows a quasi-periodic oscillation with a frequency that depends on the cooling time. Interestingly, from the observational point of view, no periodic signature in accretion shocks has been found yet $[6,7]$. By considering a more complex geometry for the magnetic field [8] (see Bonito, this volume) or by taking radiative transfer into account (see de Sá, this volume), the periodic pattern could be suppressed.

Here, we do not consider such possibilities, but we explore the evolution of an accretion shock in the simple regime of optically thin radiation losses and a uniform magnetic field. Instead, we simulate the system in more dimensions, also introducing perturbations in the system in order to study their effects on the structure and evolution of the post-shock region.

\section{Numerical approach}

We use the PLUTO code [9] to simulate the interior of the accretion stream in 3D. The computational domain (dashed line in the left panel of Fig. 1) consists of the infalling gas, the hot slab, and part of the chromosphere. We solve the magneto-hydronamic equations taking optically thin cooling and magnetic-field-oriented thermal conduction into account. Our box spans $x, y=\left[0.0,0.5 \times 10^{-3}\right] R_{\odot}$ in the horizontal directions and $z=\left[0.0,7.7 \times 10^{-2}\right] R_{\odot}$ in the vertical, and is resolved by a static grid of $[32 \times 32 \times 256]$ cells. Note that although the basic features of the dynamics can be studied in 2D, see Ref. [5], 3D simulations are necessary to provide consistent data for post-processing, part of our future work. 

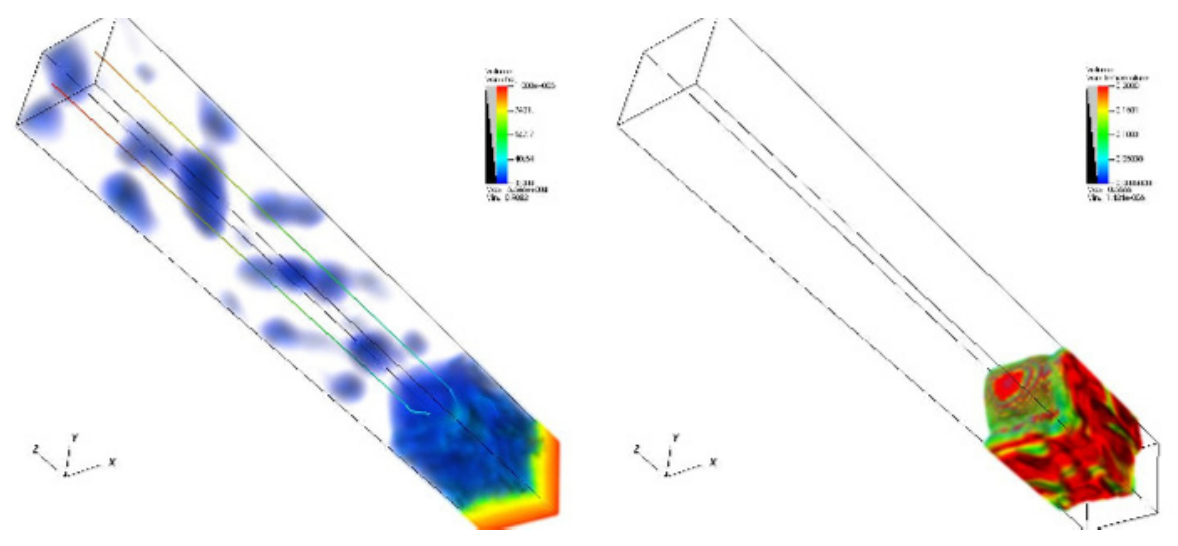

Figure 2. Volume plots of the density (left) and temperature (right) from a snapshot of the accretion shock for the case of a weak value of the magnetic field: $B_{0}=30 \mathrm{G}$. From top to bottom, the clumpy accretion stream, the post-shock region, and the chromosphere (compare also with the left panel of Fig. 1). Regions with low densities/temperatures are transparent due to visualization purposes. Magnetic fieldlines are also shown on the left panel. The legends are in code units.

Initially, at the upper part of the domain, we prescribe a uniform flow of number density $n_{\text {acc }}=$ $2 \cdot 10^{11} \mathrm{~cm}^{-3}$ and velocity $v_{\text {acc }}=500 \mathrm{~km} \mathrm{~s}^{-1}$. These conditions are specified at the top boundary too, on which we also introduce clumps of random size and location throughout the simulation time. Their density follows the gaussian distribution with the peak value set at $n_{\mathrm{clm}}=10^{12} \mathrm{~cm}^{-3}$ and standard deviation equal to $10 \%-30 \%$ of the box width $\left(\sim 10^{2}-10^{3} \mathrm{~km}\right)$. For the initial conditions at the lower part of the domain, we specify an isothermal chromosphere with $T_{\mathrm{chr}}=10000 \mathrm{~K}$ that is in equilibrium with the imposed constant gravity (the protostar is assumed of solar mass and radius). In addition to that, on the bottom boundary we also add an acoustic energy flux $\left(F_{\mathrm{chr}}=5 \times 10^{9} \mathrm{erg} \mathrm{cm}^{-2} \mathrm{~s}^{-1}\right)$ that is periodic in space $(\sim 5000 \mathrm{~km})$ and time $(\sim 5 \mathrm{~min})$. These values are close to solar observations. Finally, the left and right boundaries of the computational domain are periodic. The initial magnetic field, $B_{0}$, is considered uniform in the vertical direction. During evolution we assume that the plasma is always fully ionized and we incorporate the radiation energy loss term, $\Lambda$, in a tabulated form as shown in the right panel of Fig. 1 [4] (the cooling function is set to zero in the chromosphere).

We perform numerical simulations for three models: a) $B_{0}=30 \mathrm{G}$, b) $B_{0}=100 \mathrm{G}$, and c) $B_{0}=$ $300 \mathrm{G}$. These magnetic field values are chosen in order to explore plasma- $\beta$ values around unity (for the post-shock region), a variable that plays a critical role in the dynamics of accretion shocks. The three models considered correspond, equivalenty, to a) $\beta \gtrsim 1$, b) $\beta \simeq 1$, and c) $\beta \lesssim 1$. Moreover, note that the magnetic field on the protostellar surface is measured to be on the order of kG (see JohnsKrull, Gregory, this volume). Nonetheless, as explained later, for magnetic fields stronger than $300 \mathrm{G}$ the dynamics of the accretion shock is very similar to the case $B_{0}=300 \mathrm{G}$ and hence we do not need to consider additional models. Consequently, model c) is probably the most relevant simulation for young stars.

\section{Results}

We start with the presentation of model a) in which the plasma- $\beta$ in the post-shock region is larger than unity, and consequently the overall behavior is almost hydrodynamic. The clumps in the stream, 

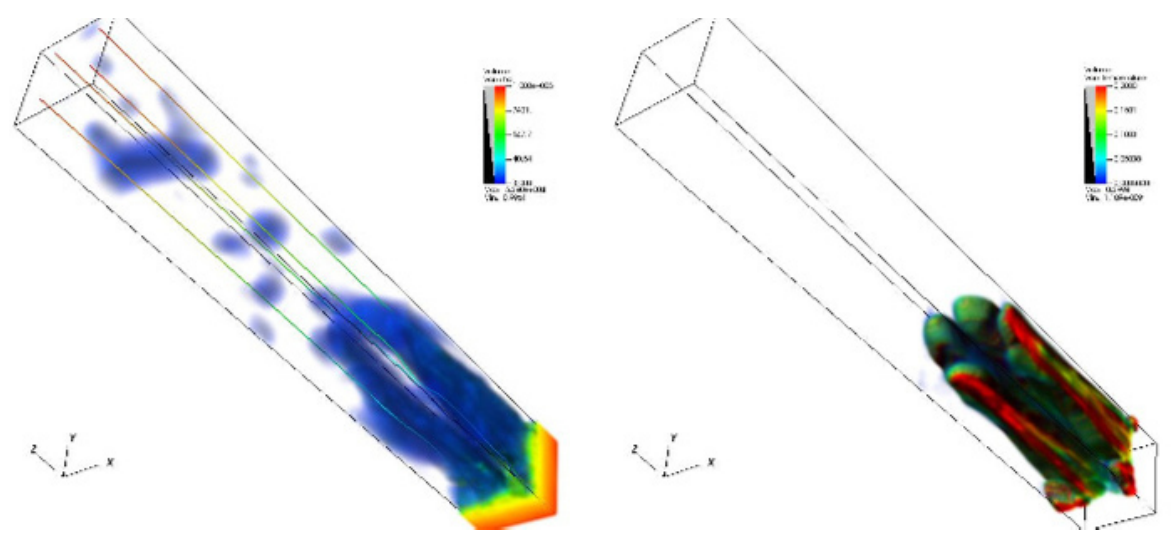

Figure 3. Same as Fig. 2 but a magnetic field of $B_{0}=100 \mathrm{G}$. The post-shock region here consists of elongated vertical structures.
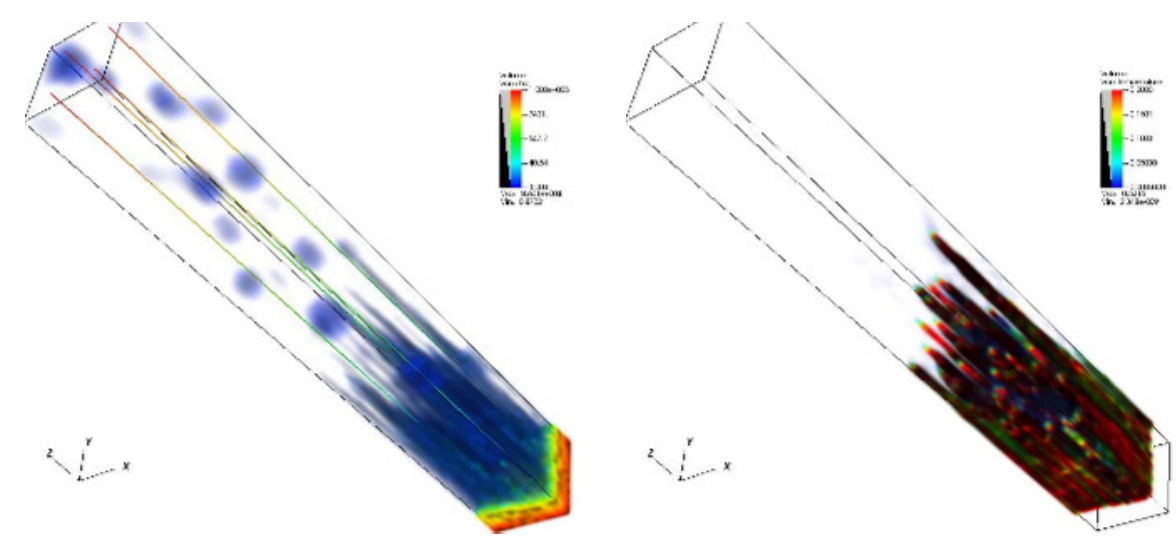

Figure 4. Same as Fig. 2 but for stronger magnetic field: $B_{0}=300 \mathrm{G}$. The post-shock region consists of fibrils that form and collapse quasi-periodically and independently of their neighbors. For even higher values of the magnetic field, the dynamics is expected to be similar.

as well as the chromospheric variability, perturb the homogeneity of the hot slab. This leads to chaotic motion and plasma mixing in the post-shock region as it can be seen by the small inhomogeneities in Fig. 2. Nevertheless, the system retains globally the formation-collapse cycle of the reverse shock, being in agreement with the $1 \mathrm{D}$ studies. This is mainly because the large post-shock sound speed smooths out, to some degree, the effects of perturbations.

The post-shock region of model $b$ ), that has a stronger magnetic field strength than a), consists of vertically elongated structures, see Fig. 3 . Since the plasma- $\beta$ there is around unity, the field lines resist the deformation that is caused by the local pressure gradients. In turn, the horizontal motion of the post-shock plasma is limited and the material gets trapped vertically. This effect disrupts the global character of the oscillation and the system has no longer a coherent behavior.

For a plasma- $\beta$ in the hot slab that is smaller than unity, i.e. model c), the magnetic field dominates the dynamics. As a result, it can effectively confine the plasma within flux tubes. Thus, material is 
constrained to move only vertically and cannot interact with its neighbors. This leads to a post-shock region that consists of fibrils, see Fig. 4. Each fibril is evolving independently, having a quasi-periodic oscillatory behavior as found in the 1D studies. As long as they form and collapse in phase, the system will exhibit a periodic behavior. However, due to the introduced perturbations, the fibrils are quickly brought out of phase. Consequently, the overall emission that results by adding up the individual contributions will not show any periodic patterns [5]. Finally, we point out that the size of the fibrils does not depend on the type of the perturbation but only on the plasma- $\beta$ value of the post-shock region [5].

\section{Conclusions}

Our study suggests that even in the simple configuration of a uniform magnetic field, the post-shock region can become highly structured. We show that there is a strong dependence on the plasma- $\beta$ value which has observational relevance to its time-dependent emission signature. Future work will focus on post-processing these data (see Ibgui, this volume) in order to create synthetic spectra and compare with observations.

\section{Acknowledgements}

This work was supported by the ANR STARSHOCK project (ANR-08-BLAN-0263-07-2009/2013) and was granted access to the HPC resources of CINES under the allocation 2012-c2012046943 and 2013-c2013046943 made by GENCI (Grand Equipement National de Calcul Intensif).

\section{References}

[1] C. Argiroffi, A. Maggio, G. Peres, A\&A, 465, L5 (2007), arXiv: astro-ph/0701765

[2] G.G. Sacco, C. Argiroffi, S. Orlando, A. Maggio, G. Peres, F. Reale, A\&A, 491, L17 (2008), 0810.0192

[3] A.V. Koldoba, G.V. Ustyugova, M.M. Romanova, R.V.E. Lovelace, MNRAS, 388, 357 (2008), 0803.2020

[4] S. Orlando, G.G. Sacco, C. Argiroffi, F. Reale, G. Peres, A. Maggio, A\&A, 510, A71 (2010), 0912. 1799

[5] T. Matsakos, J.P. Chièze, C. Stehlé, M. González, L. Ibgui, L. de Sá, T. Lanz, S. Orlando, R. Bonito, C. Argiroffi et al., A\&A, 557, 69 (2013), 1307.5389

[6] J.J. Drake, P.W. Ratzlaff, J.M. Laming, J. Raymond, ApJ, 703, 1224 (2009)

[7] H.M. Günther, N. Lewandowska, M.P.G. Hundertmark, H. Steinle, J.H.M.M. Schmitt, D. Buckley, S. Crawford, D. O’Donoghue, P. Vaisanen, A\&A, 518, A54 (2010), 1005.1885

[8] S. Orlando, R. Bonito, C. Argiroffi, F. Reale, G. Peres, M. Miceli, T. Matsakos, C. Stehlé, L. Ibgui, L. de Sá et al., A\&A, submitted (2013)

[9] A. Mignone, G. Bodo, S. Massaglia, T. Matsakos, O. Tesileanu, C. Zanni, A. Ferrari, ApJS, 170, 228 (2007), arXiv: astro-ph/0701854 
